\title{
Conocimientos sobre plantas medicinales en seis comunidades Ch'oles de Tacotalpa, Tabasco
}

\section{Knowledge of medicinal plants in six Ch'oles communities in Tacotalpa, Tabasco}

\author{
VILLEGAS-RAMÍREZ, María Isabel†*, MORALES-VALENZUELA, Guadalupe, DE LOS SANTOS- \\ RUIZ, Cynthia Paola y GÓMEZ-SÁNTIZ, Pedro
}

Licenciatura en Salud Intercultural, Universidad Intercultural del Estado de Tabasco. Gregorio Méndez 6, Centro, 86870 Tacotalpa, Tab.

\author{
ID 1 ${ }^{\text {er }}$ Autor: María Isabel, Villegas-Ramírez / ORC ID: 0000-0001-8912-5261, CVU CONACYT ID: 867564 \\ ID $1^{\text {er }}$ Coautor: Guadalupe, Morales-Valenzuela / ORC ID: 0000-0003-4289-5415, CVU CONACYT ID: 37866 \\ ID $2^{\text {do }}$ Coautor: Cynthia Paola, De Los Santos-Ruiz / ORC ID: 0000-0003-2743-9200, CVU CONACYT ID: 1015872 \\ ID $3^{\text {er }}$ Coautor: Pedro, Gómez-Sántiz
}

DOI: $10.35429 / J O C S .2019 .19 .6 .1 .6$

Recibido 29 de Abril, 2019; Aceptado 27 de Junio, 2019

\begin{abstract}
Resumen
Los Ch'oles de Tacotalpa, Tabasco; poseen conocimientos y prácticas sobre sistemas de salud tradicionales que comparten entre sí. Son bastos los saberes sobre Medicina Tradicional Mexicana (MTM) y de ello se sirven para atender su salud. Este trabajo tuvo como objetivo identificar los conocimientos que la población de seis comunidades tiene sobre las plantas medicinales y sus usos. Fue un estudio cuantitativo realizado durante enero-agosto de 2019. El instrumento utilizado fue una entrevista de seis categorías de preguntas, participaron 143 habitantes a partir de un muestreo sistemático, incluyendo jóvenes y adultos, hombres y mujeres. Como parte de los resultados, se identificaron 69 plantas medicinales utilizadas para diversos padecimientos que pertenecen a siete grupos de enfermedades: inmunológicos, gastrointestinales, respiratorios, infecciones de la piel, síndromes de filiación cultural, osteomusculares y del sistema nervioso. Se registraron 28 plantas medicinales en Buenos Aires, 14 en Noypac, 49 en Oxolotán, 27 en Puxcatán, 22 en Guayal y 17 en Libertad. Las plantas con mayor mención son el maguey, sábila, albahaca, ruda y epazote; algunas de estas son introducidas a la región, como la moringa, manzanilla y azafrán. Las enfermedades en las que son utilizados son principalmente tos, heridas, infección, dolor de estómago, mal de ojo y diarrea. Existe una variedad de conocimientos y prácticas relativas a la medicina tradicional que vale la pena identificar, sistematizar y compartir al interior y exterior de las comunidades, que permita el reconocimiento de los saberes comunitarios y tradicionales.
\end{abstract}

Enfermedades, Medicina tradicional mexicana

\begin{abstract}
The Ch'oles of Tacotalpa, Tabasco; have knowledge and practices about traditional health systems that they share with each other. Knowledge about Traditional Mexican Medicine (TMM) is enough and it is used to take care of their health. This work had the purpose to identify the knowledge that the population of six communities have about medicinal plants and their uses. It was a quantitative study carried out during January-August 2019. An interview of six categories of questions was the instrument used, involving 143 inhabitants from systematic sampling, including youth and adults, men and women. As part of the results, 69 medicinal plants used for various conditions belonging to seven groups of diseases were identified: immunological, gastrointestinal, respiratory, skin infections, cultural affiliation syndromes, osteomuscular and nervous system. 28 medicinal plants were registered in Buenos Aires, 14 in Noypac, 49 in Oxolotán, 27 in Puxcatán, 22 in Guayal and 17 in Libertad. The plants with the greatest mention are maguey, sábila, basil, rue and epazote; some of these are introduced to the region, such as moringa, chamomile and saffron crocus. The diseases in which they are mainly used are cough, injuries, infection, stomachache, evil eye and diarrhea. There is a variety of knowledge and practices relating to traditional medicine that are worth identifying, systematizing and sharing inside and outside the communities, allowing the recognition of community and traditional knowledge.
\end{abstract}

Diseases, Traditional mexican medicine

Citación: VILLEGAS-RAMÍREZ, María Isabel, MORALES-VALENZUELA, Guadalupe, DE LOS SANTOS-RUIZ,

Cynthia Paola, GÓMEZ-SÁNTIZ, Pedro. Conocimientos sobre plantas medicinales en seis comunidades Ch'oles de Tacotalpa, Tabasco. Revista de Sociología Contemporánea. 2019. 6-19: 1-6.

\footnotetext{
* Correspondencia del Autor (correo electrónico: is_villegas@ @otmail.com)

$\uparrow$ Investigador contribuyendo como primer autor.
} 


\section{Introducción}

Los pueblos originarios de México son poseedores de una vasta gama de conocimientos sobre los recursos bioculturales, dentro de ellos se incluye las plantas medicinales, propias de cada región y de acuerdo a la cosmovisión de cada grupo cultural (Villegas et al. 2019).

Los Ch'oles del estado de Tabasco, específicamente los que pertenecen al municipio de Tacotalpa, tienen prácticas que derivan de sus saberes sobre las plantas locales que actúan sobre padecimientos que se diferencian de la nosología científica y que son parte de los conocimientos de la Medicina Tradicional Mexicana (MTM)

$\mathrm{Al}$ respecto, la ley Marco en materia de Medicina Tradicional define a esta como procesos de atención a la salud que tienen como base conocimientos sobre la salud y enfermedad cimentados en una cosmovisión precolombina y al mismo tiempo enriquecida por las culturas españolas, portuguesas, africanas, pero también de la ciencia médica (Almaguer, 2009).

Con base a estos planteamientos, es importante acceder a los conocimientos que las poblaciones Ch'oles del municipio de Tacotalpa tienen sobre plantas medicinales, sus usos y adquisición de dicho conocimiento.

Desde la medicina científica, se han realizado investigaciones (colocar alguna cita) para conocer los ingredientes activos de las plantas que refieren propiedades curativas, pero se establecen con la finalidad de la comprobación científica y el uso comercial o bien como propuesta de la salud pública para el control de la salud, no con el interés del reconocimiento de los terapeutas tradicionales que tienen una experiencia empírica.

Se requieren entonces estudios más etnobotánicos o etnofarmacológicos, investigaciones que al mismo tiempo que puedan inventariar o caracterizar el uso y propiedades de las plantas, puedan describir los aspectos culturales y procesos sociales en torno a estos conocimientos.
Al respecto se han establecido algunos estudios por Santos-Padrón et al. (2005), ChanQuijano et al. (2015), Pérez et al. (2015) y Villegas et al. (2018 y 2019) buscando tener un acercamiento a los conocimientos y prácticas tradicionales sobre las plantas medicinales de los pueblos rurales e indígenas de la Sierra Sur Tabasqueña y especialmente de los Ch'oles.

Se entiende por conocimientos tradicionales en materia de la medicina tradicional, como el "conjunto de prácticas y saberes colectivos de los pueblos indígenas referidos a la biodiversidad, a la saludenfermedad y al manejo de los recursos orientados al bienestar comunitario" (Almaguer, 2099:5) Por otro lado, las plantas medicinales pertenecen al grupo de medicamentos herbarios que reconoce la OMS (2018) contienen principios activos y son usados para el mantenimiento de la salud, así como para la prevención, el diagnóstico, la mejora o el tratamiento de enfermedades físicas o mentales.

Es así que este trabajo se convierte en un documento importante en la medida que documenta la evidencia de los conocimientos que poseen los pobladores de al menos seis de las 31 comunidades con más de $40 \%$ de población indígena del municipio de Tacotalpa; poder sistematizar y devolver la información, así como compartir en espacios académicos para su reconocimiento.

\section{Métodos}

La selección de las comunidades participantes se hizo con base en la categorización de localidades indígenas que establece la Comisión Nacional para los Pueblos Indígenas (CDI, 2010), considerando que existiera población hablante Ch'ol. Este trabajo es de corte cuantitativo y se llevó a cabo durante los meses de enero a agosto de 2019, realizando un solo corte en cada comunidad participante. Se aplicaron 143 entrevistas estructuradas a partir de un muestreo sistemático, incluyendo jóvenes y adultos, hombres y mujeres en seis comunidades: Buenos Aires Guayal, Libertad, Noypac, Puxcatán y Oxolotán. El instrumento utilizado consistió en una entrevista donde se incluyen 4 categorías: 1 ) Conocimientos y usos de las plantas, 2) utilización de las plantas, 3) enfermedades en que se utilizan las plantas y 4) adquisición del conocimiento. 


\section{Resultados y discusión}

1. Características de las localidades.

De acuerdo a información de la CDI (2010) la población que vive en las seis comunidades es de 5709 habitantes, de los cuales $75.16 \%$ es población indígena. La mayoría de las comunidades tiene un alto grado de marginación (ver Tabla 1).

\begin{tabular}{|l|l|l|r|r|r|}
\hline Localidad & $\begin{array}{c}\text { Tipo de } \\
\text { localidad }\end{array}$ & \multicolumn{1}{c}{$\begin{array}{c}\text { Grado de } \\
\text { Marginación }\end{array}$} & $\begin{array}{c}\text { Población } \\
\text { total }\end{array}$ & $\begin{array}{c}\text { Población } \\
\text { Indígena }\end{array}$ & $\begin{array}{c}\text { Porcentaje } \\
\text { de } \\
\text { población } \\
\text { indígena }\end{array}$ \\
\hline $\begin{array}{l}\text { Buenos } \\
\text { Aires }\end{array}$ & $\begin{array}{l}\text { Loc. de } \\
40 \% \text { y más }\end{array}$ & Alto & 253 & 236 & 93.28 \\
\hline Guayal & $\begin{array}{l}\text { Loc. de } \\
40 \% \text { y más }\end{array}$ & Alto & 954 & 947 & 99.27 \\
\hline Libertad & $\begin{array}{l}\text { Loc. de } \\
40 \% \text { y más }\end{array}$ & Alto & 1,042 & 1,028 & 98.66 \\
\hline Noypac & $\begin{array}{l}\text { Loc. de } \\
40 \% \text { y más }\end{array}$ & Alto & 286 & 278 & 97.20 \\
\hline Oxolotán & $\begin{array}{l}\text { Loc. de } \\
\text { interés de }\end{array}$ & Medio & 1,886 & 577 & 30.59 \\
\hline Puxcatán & $\begin{array}{l}\text { Loc. de } \\
40 \% \text { y más }\end{array}$ & Alto & 1,288 & 1,225 & 95.11 \\
\hline
\end{tabular}

Tabla 1 Características de las comunidades estudiadas

2. Conocimiento de las plantas medicinales y sus usos.

Se identificaron un total de 69 plantas medicinales en el total de las comunidades, de las cuales 28 fueron mencionadas en Buenos Aires, 20 en Noypac, 49 en Oxolotán, 27 en Puxcatán, 22 en Guayal y 17 en Libertad. En el gráfico 1 se muestra de acuerdo a la comunidad.

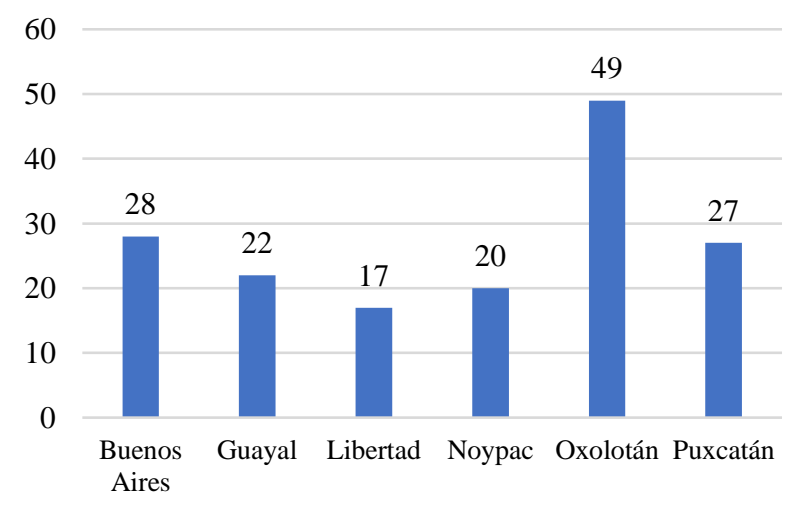

Gráfico 1 Número de Plantas medicinales mencionadas por comunidad

Las plantas con mayor mención en las 6 comunidades son el maguey, sábila, hierbabuena, manzanilla, y epazote (Tabla 1); otras son igual mencionadas con ausencia en al menos una comunidad: Albahaca, llantén, ruda, zacate limón, guayaba, belladona, mala madre, cempasúchil y el guácimo.

\begin{tabular}{|c|c|c|c|c|c|c|c|}
\hline Nombre común & $\begin{array}{l}\text { Nombre } \\
\text { científico }\end{array}$ & BA & Gua & Lib. & Noy. & Oxo. & Pux. \\
\hline Maguey & $\begin{array}{l}\text { Tradescantia } \\
\text { Spathacea }\end{array}$ & $\mathrm{X}$ & $\mathrm{x}$ & $\mathrm{x}$ & $\mathrm{X}$ & $\mathrm{X}$ & $\mathrm{X}$ \\
\hline Epazote & $\begin{array}{l}\text { Chenopodium } \\
\text { ambrosioides } L \text {. }\end{array}$ & $\mathrm{X}$ & $\mathrm{X}$ & $\mathrm{X}$ & $\mathrm{X}$ & $\mathrm{X}$ & $\mathrm{X}$ \\
\hline Albahaca & $\begin{array}{l}\text { Ocimum } \\
\text { basilicum L. }\end{array}$ & $\mathrm{X}$ & 0 & $\mathrm{X}$ & $\mathrm{X}$ & $\mathrm{X}$ & $\mathrm{X}$ \\
\hline Llantén & $\begin{array}{l}\text { Plantago major } \\
L \text {. }\end{array}$ & $\mathrm{X}$ & 0 & 0 & $\mathrm{X}$ & $\mathrm{X}$ & $\mathrm{X}$ \\
\hline Sábila & Aloe vera $L$. & $\mathrm{X}$ & $\mathrm{X}$ & $\mathrm{X}$ & $\mathrm{X}$ & $\mathrm{X}$ & $\mathrm{X}$ \\
\hline Ruda & $\begin{array}{l}\text { Ruta graveolens } \\
\text { L. }\end{array}$ & $\mathrm{X}$ & 0 & $\mathrm{X}$ & $\mathrm{X}$ & $\mathrm{X}$ & $\mathrm{X}$ \\
\hline Zacate Limón & $\begin{array}{l}\text { Cymbopogon } \\
\text { citratus }\end{array}$ & $\mathrm{X}$ & $\mathrm{X}$ & 0 & $\mathrm{X}$ & $\mathrm{X}$ & $\mathrm{X}$ \\
\hline Hierbabuena & Mentha spicata & $\mathrm{X}$ & $\mathrm{X}$ & $\mathrm{X}$ & $\mathrm{X}$ & $\mathrm{X}$ & $\mathrm{X}$ \\
\hline Guayaba & $\begin{array}{l}\text { Psidium } \\
\text { guajava L. }\end{array}$ & $\mathrm{X}$ & $\mathrm{X}$ & 0 & $\mathrm{X}$ & $\mathrm{X}$ & $\mathrm{X}$ \\
\hline Belladona & $\begin{array}{l}\text { Kalanchoe } \\
\text { flammea Stapf }\end{array}$ & $\mathrm{X}$ & 0 & 0 & $\mathrm{X}$ & $\mathrm{X}$ & $\mathrm{X}$ \\
\hline Curatodo o mala madre & $\begin{array}{l}\text { Chlorophytum } \\
\text { Comosum }\end{array}$ & 0 & 0 & $\mathrm{X}$ & $\mathrm{X}$ & $\mathrm{X}$ & $\mathrm{X}$ \\
\hline Oreganón & $\begin{array}{l}\text { Lippia } \\
\text { graveolens } \\
\text { Kunth } \\
\end{array}$ & $\mathrm{X}$ & $\mathrm{X}$ & $\mathrm{X}$ & $\mathrm{X}$ & $\mathrm{X}$ & $\mathrm{X}$ \\
\hline Cempasúchil & $\begin{array}{l}\text { Tagetes erecta } \\
\mathrm{L} \text {. }\end{array}$ & 0 & $\mathrm{X}$ & $\mathrm{X}$ & $\mathrm{X}$ & $\mathrm{X}$ & $\mathrm{X}$ \\
\hline Manzanilla & $\begin{array}{l}\text { Matricaria } \\
\text { chamomilla L. }\end{array}$ & $\mathrm{X}$ & $\mathrm{X}$ & $\mathrm{X}$ & $\mathrm{X}$ & $\mathrm{X}$ & $\mathrm{X}$ \\
\hline Guácimo & $\begin{array}{l}\text { Guazuma } \\
\text { ulmifolia Lam }\end{array}$ & $\mathrm{X}$ & $\mathrm{X}$ & 0 & 0 & 0 & $\mathrm{X}$ \\
\hline
\end{tabular}

Tabla 2 Matriz de presencia y ausencia de las 15 plantas más mencionadas en las seis comunidades

Cabe mencionar que la manzanilla no es propia de la región, pero igual se tiene el conocimiento de sus propiedades curativas, al igual que la moringa y el azafrán, plantas que han sido introducidas a la región. Los usos que les otorgan son en padecimientos que pertenecen a nueve grupos: inmunológicos, gastrointestinales, respiratorios, infecciones de la piel, síndromes de filiación cultural, osteomusculares, crónico-degenerativos, del sistema nervioso y reproductor. tal y como se muestra en la Tabla 3.

\begin{tabular}{|ll|l|}
\hline \multicolumn{2}{|c|}{$\begin{array}{l}\text { Grupo de } \\
\text { padecimientos }\end{array}$} & \multicolumn{1}{c|}{ Padecimientos } \\
\hline 1. & Inmunológicos & $\begin{array}{l}\text { Infecciones, alergias, conjuntivitis, } \\
\text { fiebre }\end{array}$ \\
\hline 2. & Gastrointestinales & $\begin{array}{l}\text { Dolor de estómago, cólicos, } \\
\text { parásitos, diarrea, gastritis, } \\
\text { estreñimiento, colitis, vómito }\end{array}$ \\
\hline 3. & Cutáneos & $\begin{array}{l}\text { Comezón, rozaduras, quemaduras, } \\
\text { varicela, heridas, herpes }\end{array}$ \\
\hline 4. & Respiratorios & $\begin{array}{l}\text { Tos, dolor de garganta, asma, } \\
\text { sinusitis, gripe }\end{array}$ \\
\hline 5. & $\begin{array}{l}\text { Síndromes de } \\
\text { filiación cultural }\end{array}$ & $\begin{array}{l}\text { Mal aire, espanto, calentamiento de } \\
\text { cabeza, vista caliente, mal de ojo, } \\
\text { caída de mollera }\end{array}$ \\
\hline 6. & Osteomusculares & $\begin{array}{l}\text { Torceduras, dolor de pie, dolores del } \\
\text { cuerpo, reumas }\end{array}$ \\
\hline 7. & $\begin{array}{l}\text { Crónico- } \\
\text { degenerativos y } \\
\text { dislipidemias }\end{array}$ & $\begin{array}{l}\text { Diabetes, presión alta, triglicéridos, } \\
\text { colesterol }\end{array}$ \\
\hline 8. & Sistema nervioso & $\begin{array}{l}\text { Insomnio, depresión, tensión, } \\
\text { nerviosismo, estrés }\end{array}$ \\
\hline 9. & $\begin{array}{l}\text { Sistema } \\
\text { reproductor }\end{array}$ & Menstruación, quistes \\
\hline & & \\
\hline
\end{tabular}

Tabla 3 Padecimientos en los que utilizan plantas medicinales 
Ahora bien, las plantas con mayor número de menciones fueron el maguey $(69.07 \%)$ ruda $(28.06 \%)$, epazote $(26.8 \%)$, sábila $(23.16 \%)$, albahaca (22.07) y llantén (17.27\%).

\section{Utilización de plantas}

La OMS (2008) reconoce que más del $80 \%$ de la población a nivel mundial ocupa las plantas medicinales y otros recursos naturales para la atención de su salud y valida su eficacia. Lo cierto es que en lugares donde no se cuenta con personal de salud no espacios para su atención, las poblaciones se han mantenido precisamente por la dieta local y las plantas que tienen a su alcance.

Un hecho es conocer el uso de las plantas y otra es ocuparla para el autoconsumo como parte del proceso de salud-enfermedad-atención. Generalmente se toma en cuenta el conocimiento que se tiene de las propiedades curativas, pero no se pregunta si efectivamente recurren a tal planta para el tratamiento de una enfermedad. Se encontró un total de 121 personas que afirmaron consumir las plantas para tratarse algún tipo de enfermedad, siendo las comunidades de Noypac y Oxolotán, las que más consumen con un total de 26 personas cada una, de acuerdo a los participantes. En este sentido, en el Gráfico 2 se muestra el número de plantas utilizadas por comunidad. Como puede apreciarse, en Puxcatán casi se usa la misma cantidad de plantas conocidas.

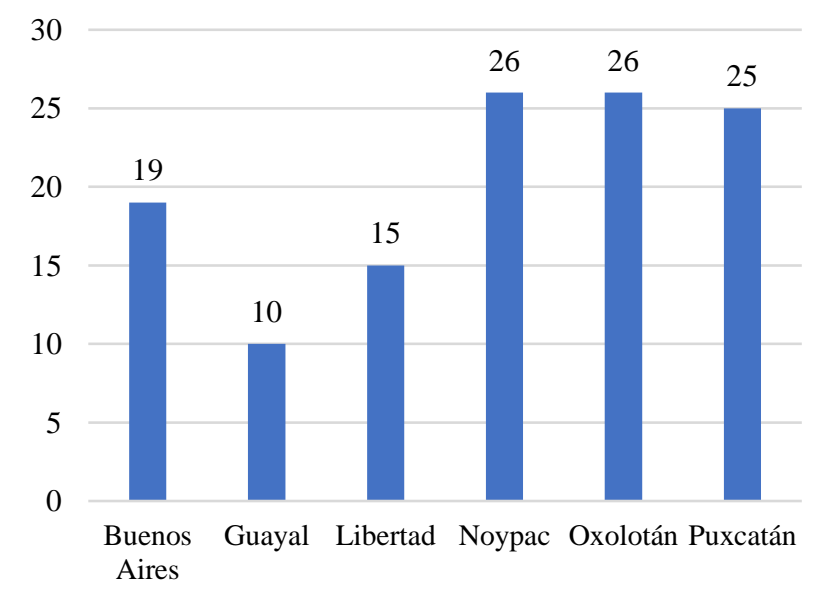

Gráfico 2 Número de plantas medicinales utilizadas por comunidad
Es de notar que, aunque en algunas comunidades se mencionaron un buen número de plantas y que conocen para qué sirven, no necesariamente las han consumido, como el caso de Buenos Aires, Guayal, Libertad y Oxolotán (Tabla 4).

\begin{tabular}{|l|r|r|r|}
\hline Comunidad & \multicolumn{1}{c}{$\begin{array}{c}\text { Número } \\
\text { de plantas } \\
\text { conocidas }\end{array}$} & \multicolumn{1}{c|}{$\begin{array}{c}\text { Número } \\
\text { de plantas } \\
\text { utilizadas }\end{array}$} & Diferencias \\
\hline $\begin{array}{l}\text { Buenos } \\
\text { Aires }\end{array}$ & 28 & 19 & 9 \\
\hline Guayal & 22 & 10 & 12 \\
\hline Libertad & 17 & 15 & 2 \\
\hline Noypac & 20 & 26 & -6 \\
\hline Oxolotán & 49 & 26 & 23 \\
\hline Puxcatán & 27 & 25 & 8 \\
\hline
\end{tabular}

Tabla 4 Comparación del número de plantas conocidas y utilizadas

En el caso de Noypac se mencionaron más plantas utilizadas que conocidas. Lo anterior difiere de lo encontrado por SantosPadrón et al. (2005): que la población de Tacotalpa consume 55\% de medicamentos y el otro 55\% de remedios caseros, ocupando así el segundo lugar (después de Jalpa de Méndez) en el consumo de plantas medicinales, en el estado de Tabasco.

4. Adquisición del conocimiento de las plantas.

Como los demás aprendizajes, el conocimiento que poseen las comunidades obedece a la vida cotidiana, a los recursos con los que cuentan las poblaciones para la atención de la salud: la biodiversidad, esto es, las plantas, animales y minerales. Se encontraron 7 mecanismos de cómo se adquiere el conocimiento sobre las propiedades y usos de las plantas. Las más mencionadas fueron la enseñanza de sus abuelos o padres y los médicos tradicionales, lo que deja ver que, en cada comunidad, hay presencia de estos y las personas recurren a sus servicios; sin embargo, también se mencionó el aprendizaje mediante la experiencia empírica, lectura de libros relacionados, receta por un médico alópata y capacitación en talleres (Tabla 5). 


\begin{tabular}{|ll|l|l|l|l|l|l|}
\hline & BA & Gua & Lib. & Noy. & Oxo. & Pux. \\
\hline 1. & $\begin{array}{l}\text { Familiar } \\
\text { (abuelo, } \\
\text { padres, pareja) }\end{array}$ & $\mathrm{X}$ & $\mathrm{X}$ & $\mathrm{X}$ & $\mathrm{X}$ & $\mathrm{X}$ & $\mathrm{X}$ \\
\hline 2. & $\begin{array}{l}\text { Por } \\
\text { enfermedad }\end{array}$ & $\mathrm{X}$ & $\mathrm{X}$ & $\mathrm{X}$ & $\mathrm{X}$ & 0 & $\mathrm{X}$ \\
\hline 3. & $\begin{array}{l}\text { Médico } \\
\text { alópata }\end{array}$ & $\mathrm{X}$ & 0 & 0 & 0 & 0 & 0 \\
\hline 4. & $\begin{array}{l}\text { Médico } \\
\text { tradicional }\end{array}$ & $\mathrm{X}$ & $\mathrm{X}$ & $\mathrm{X}$ & $\mathrm{X}$ & $\mathrm{X}$ & $\mathrm{X}$ \\
\hline 5. & $\begin{array}{l}\text { Libros } \\
\text { (leyendo) }\end{array}$ & 0 & 0 & $\mathrm{X}$ & $\mathrm{X}$ & $\mathrm{X}$ & 0 \\
\hline 6. & $\begin{array}{l}\text { Capacitaciones } \\
\text { sobre plantas }\end{array}$ & 0 & 0 & 0 & 0 & $\mathrm{X}$ & 0 \\
\hline 7. & $\begin{array}{l}\text { Sólo, } \\
\text { manera } \\
\text { empírica }\end{array}$ & 0 & $\mathrm{X}$ & $\mathrm{X}$ & 0 & $\mathrm{X}$ & $\mathrm{X}$ \\
\hline
\end{tabular}

Tabla 5 Formas de adquisición del conocimiento de las plantas medicinales

\section{Conclusiones}

Existe una variedad de conocimientos y prácticas relativas a la medicina tradicional que vale la pena identificar, sistematizar y compartir al interior y exterior de las comunidades, que permita el reconocimiento de los saberes comunitarios y tradicionales. La importancia de que la población aun mantenga estos conocimientos y prácticas radica en cuatro hechos:

1) Se mantienen los mecanismos de transmisión de los saberes tradicionales y locales.

2) Representan los recursos al alcance para tratar las enfermedades en donde el sistema de salud actual no cuenta con la infraestructura y recursos humanos para la atención a la salud.

3) Permite el empoderamiento sobre el cuerpo $\mathrm{y}$ el bienestar en tanto constituye una posibilidad de decidir sobre la mejor manera de abordar la salud mientras existan otras alternativas en el proceso de salud/enfermedad/atención.

4) Aún se cuentan con los recursos naturales y culturales, es decir, las plantas las cuales utilizan son cultivados o se recolectan por ser silvestres, estas plantas simbolizan los conocimientos ancestrales y culturales que hacen un andamiaje biocultural.

\section{Agradecimientos}

A las comunidades de la región Ch'ol de Tacotalpa quienes aceptaron participar en el proyecto. Red Temática sobre Patrimonio Biocultural del Consejo Nacional de Ciencia y Tecnología (CONACYT) por el apoyo para el trabajo de campo en la realización de este proyecto.

\section{Referencias}

Alejos, J. \& Martínez, N. E. (2007). Ch'oles. Primera edición. México: Comisión Nacional para el Desarrollo de los Pueblos Indígenas.

Almaguer, J. A. (2009). Ley marco en materia de medicina tradicional. México: Comisión de Salud del Parlamento Latinoamericano.

Chan-Quijano, J., Villanueva-López, G., Ramos-Muñoz, D. E. \& Juárez-García, A. (2014). Traditional use of the flora in the "el chile" the Pomoca Ejido, Tacotalpa, Tabasco, Mexico. Gaia Scientia. (1981-1268, 172-188) recuperado 30 de agosto de 2019 en https://www.researchgate.net/publication/27059 2808

Comisión Nacional para el Desarrollo De los pueblos indígenas (CDI). (2006). Regiones indígenas de México. México: CDI.

Comisión Nacional para el Desarrollo De los pueblos indígenas (CDI). (2010). Catálogo de localidades indígenas. México: CDI

Espinosa, M. J., Centurión H. D., Mayo, M. A. \& Velázquez, M. J. R. (2017). Plantas aromáticas y medicinales tropicales con potencial actividad antimicrobiana. México: Universidad Juárez Autónoma de Tabasco.

Gómez, E. H, Germosén-Robineau, L. \& Nossin, E. (2009). Estudio etnofarmacológico de las plantas medicinales usadas en el Caribe colombiano. En Reyes, S. G. L. (2009). Diálogo de saberes: plantas medicinales, salud $\mathrm{y}$ cosmovisiones. Bogotá: ARFO Editores e Impresos Ltda. 135-156

Instituto Nacional de Estadística, Geografía e Informática (INEGI). (2010). Censo de d población y vivienda 2010. 
Instituto Nacional de Estadística, Geografía e Informática. (2016). Panorama sociodemográfico de Tabasco 2015. 2016.

Jorand, B. (2008). Formas de transmisión del conocimiento de la medicina tradicional en los pueblos Nahuas en el municipio de Hueyapan, sierra Norte de Puebla. Cuicuilco, 44, 181-196, septiembre-diciembre.

Morales-Valenzuela, G., Padilla-Vega, J. \& Vásquez-Dávila, M. A. (2019). Memoria biocultural de la selva. Universidad Intercultural del Estado de Tabasco y Red Temática Sobre el Patrimonio Biocultural de México-CONACYT.

OMS (2008). http://www.who.int/es/

OMS (2018). Pautas Generales para las Metodologías de Investigación y Evaluación de la Medicina Tradicional.

Pérez, V. A., Arcos, P. G., Ortiz P. G., Arcos, L. N., de los Santos, R. C. P. (2015). La tradición oral como una forma de enseñanza del uso de plantas, medicinales. Hacia un reconocimiento de la medicina tradicional en Tacotalpa, Tabasco. En moreno, U. V., Gómez, V. E. (Coords.). Investigación vinculada en las universidades interculturales de México (pp. 129-147). México: Universidad Intercultural del Estado de Tabasco y Universidad Veracruzana.

Salazar, C. E. del C., Zavala C. J., Castillo A. O. y Cámara A. R. (2014). Evaluación espacial y temporal de la vegetación de la Sierra Madrigal, Tabasco, México (1973-2003). Investigaciones Geográficas, Boletín del Instituto de Geografía, UNAM, 54, 7-23.

Santos-Padrón, H. Mier y Terán-Suárez, J., Guadalupe, M., Aguilar-Barojas, S., MedinaMedina, A. E., Torres-Valenzuela, R. (eneroagosto, 2005). Confianza de la población indígena en la atención que otorgan el médico institucional y el tradicional en el Estado de Tabasco Salud en Tabasco, 11 (1-2) 303-308.
Sosa, M. E., Padilla-Vega, J., \& Carreño-Ruiz, S. D. (2019). Los hongos comestibles y medicinales de Pomoquita, Tacotalpa, Tabasco: aspectos bioculturales para su conservación y aprovechamiento sustentable. En: En: MoralesValenzuela, G., Padilla-Vega, J. \& VásquezDávila, M. A. Memoria biocultural de la selva. Universidad Intercultural del Estado de Tabasco y Red Temática Sobre el Patrimonio Biocultural de México-CONACYT. 97-124. 78-96.

Villegas-Ramírez, M. I., Pérez-Pérez, E., NiñoHernández, A. J. \& Villega-Jiménez, W. D. (2018). Concepción de la salud y enfermedad, transmisión del conocimiento y práctica terapéutica desde la mirada de las terapeutas tradicionales de Oxolotán, Tacotalpa, Tabasco. En: Morales-Valenzuela, G., Reyes-Cruz, E. \& Contreras, C. J. R. Desafíos y perspectivas de la investigación bajo el enfoque de la interculturalidad. México: CONACYT. 65-78.

Villegas-Ramírez, M. I., Pérez-Pérez, E., NiñoHernández, A. J. Villega-Jiménez, W. D., \& Sánchez-Domínguez, H. (2019). Usos medicinales de la fauna en Oxolotán, Tacotalpa, Tabasco. En: Morales-Valenzuela, G., PadillaVega, J. \& Vásquez-Dávila, M. A. Memoria biocultural de la selva. Universidad Intercultural del Estado de Tabasco y Red Temática Sobre el Patrimonio Biocultural de México -CONACYT. 97-124. 